\title{
Preparation and Characterization of CuS-Graphene Composite
}

\author{
S.G. $\operatorname{Pan}^{1,2, *}$ \\ ${ }^{1}$ Changzhou Vocational Institute of Light Industry, Changzhou, Jiangsu, 213164, China \\ ${ }^{2}$ Key Laboratory of Soft Chemistry and Functional Materials, Nanjing University of Science and \\ Technology, Ministry of Education, Nanjing, China \\ panshugang2006@163.com \\ ${ }^{*}$ Corresponding author
}

Keywords: Graphene oxide, graphene, CuS

\begin{abstract}
A composite of CuS-graphene was prepared via a mild experimental condition, using copper nitrate, EDTA, glucose, carbon disulfide, ethylenediamine and graphene oxide as the raw materials. The composite was characterized by X-ray power diffraction, X-ray photoelectron spectroscopy, transmission electron microscopy and scanning electron microscope. In this process, graphene oxide was reduced to graphene with the formation of CuS.
\end{abstract}

\section{Introduction}

During the last years, materials scientists have been showed enough interest and made great efforts to develop and apply the transition-metal chalcogenides. Transition-metal chalcogenides can be widely used in sensors, catalyst, lithium battery; optical devices, solar cell, and so on ${ }^{[1-3]}$. Copper sulfides, an important member of the transition-metal chalcogenides, is one of the p-type compound semiconductors with narrow band gap. They were potentially important material in optical filter, superionic material, cathode material, catalyst, solarcontroller, etc ${ }^{[4,5]}$. Copper sulfides with various morphologies have been successfully fabricated involving nanoparticles, nanorods nanowires, nanotrees, nanoplates, hollows pheres, nanoflakes ${ }^{[6,7]}$.

Graphene, as its many excellent properties ${ }^{[8,9]}$, have attracted significant attention. It has been employed to as electrode material, sensors, nanoelectronics, energy storage devices, and capacitors ${ }^{[10]}$. Meanwhile, as a new carbon material, graphene can also be used to prepare the composite material. There were a lot of graphene-based composite materials. Our group has been achieved $\mathrm{Ni}_{3} \mathrm{~S}_{2} /$ Graphene, CdS/Graphene, ZnS-Graphene, and $\mathrm{Ag}_{2} \mathrm{~S}-G r a p h e n e$ composite ${ }^{[11-14]}$.

Herein, we demonstrate a facile fabrication of graphene-copper sulfides composite via a mild reaction conditions. Throughout the experiment, graphene oxide (GO) was reduced as a support material. Simultaneously, copper sulfides were attached onto the graphene sheets, and prevented the aggregation of the graphene sheets.

\section{Experimental section}

\section{Preparation of CuS-Graphene composite}

GO was prepared from natural graphite powder according to the method reported by Hummers and Offeman ${ }^{[15]}$. In a typical preparation about CuS-Graphene composite (CuS-G) ${ }^{[16]}: 20 \mathrm{mg}$ of GO was dispersed into $200 \mathrm{~mL}$ of distilled water with sonication for $45 \mathrm{~min}$. Then, $250 \mathrm{mg}$ of $\mathrm{Cu}$ $\left(\mathrm{NO}_{3}\right)_{2} \cdot 3 \mathrm{H}_{2} \mathrm{O}$ and $300 \mathrm{mg}$ of EDTA were added with stirring for $3 \mathrm{~h}$ at room temperature. Concomitantly, $0.1 \mathrm{M}$ glucose solution was added and stirred for $30 \mathrm{~min}$. afterwards, $1 \mathrm{M} \mathrm{NaOH}$ was added and stirred for $10 \mathrm{~min}$. After that, to the above system, the solution was heated to $90{ }^{\circ} \mathrm{C}$ in a water bath and kept at this temperature for $1 \mathrm{~h}$. Finally, the solution A was added and stirred for a period of time. The precipitate was collected by centrifugation, and then it was washed with distilled water and absolute ethanol several times and dried in a vacuum oven at $60{ }^{\circ} \mathrm{C}$ for $12 \mathrm{~h}$. Solution $\mathrm{A}^{[13]}$ consisted of Carbon disulfide $\left(\mathrm{CS}_{2}\right)$ and ethylenediamine aqueous solution. This solution, in a beaker, was stirred at room temperature till there was no $\mathrm{CS}_{2}$ droplet found at the 
bottom of the container. For comparison, the same method was used to synthesize CuS without GO.

\section{Characterization}

The compositions and structures of the samples were characterized by powder X-ray diffraction (XRD) using a Bruker D8 Advance diffractometor with $\mathrm{Cu} \mathrm{Ka}$ radiation $(\lambda=0.15418 \mathrm{~nm})$. The aerogel microstructure was imaged using JEOL JEM-2100 transmission electron microscopy (TEM). Scanning electron microscope (SEM, HITACHIS-4800) was used to analyze the morphology of the samples. Fourier transform infrared (FTIR) spectra of $\mathrm{KBr}$ powder pressed pellets were recorded on a Bruker VECTOR 22 spectrometer in the frequency range of 4000-450 $\mathrm{cm}^{-1}$. X-ray photoelectron spectra (XPS) were carried out on a RBD upgraded PHI-5000C ESCA system (Perkin Elmer) with Mg Ka radiation (h=1253.6 eV).

\section{Results and Discussion}

The as-prepared GO, graphene, CuS and CuS-G product were characterized by XRD. It is seen from fig. 1 that all the patterns of these two powders can be indexed accurately on the standard diffraction data of hexagonal CuS phase (JCPDS No.02-0820, space group: P63/mmc). The $2 \theta$ values of the peaks are 27.5, 29.4, 31.8, and 48.1, assigning to (101), (102), (103), and (110) reflections of hexagonal CuS, respectively. However, there is no peak of GO (001) or graphene (002) in composite. According to research reported in our previous, there are several possible causes for this phenomenon. This is because GO was reduced to graphene and the exfoliated graphene show no peak of (002). Moreover, the crystal growth of CuS between the interlayers can destroy the regular layer stacking of GO, resulting the weakening or even disappearance of the GO (001) diffraction peak ${ }^{[17,18]}$.

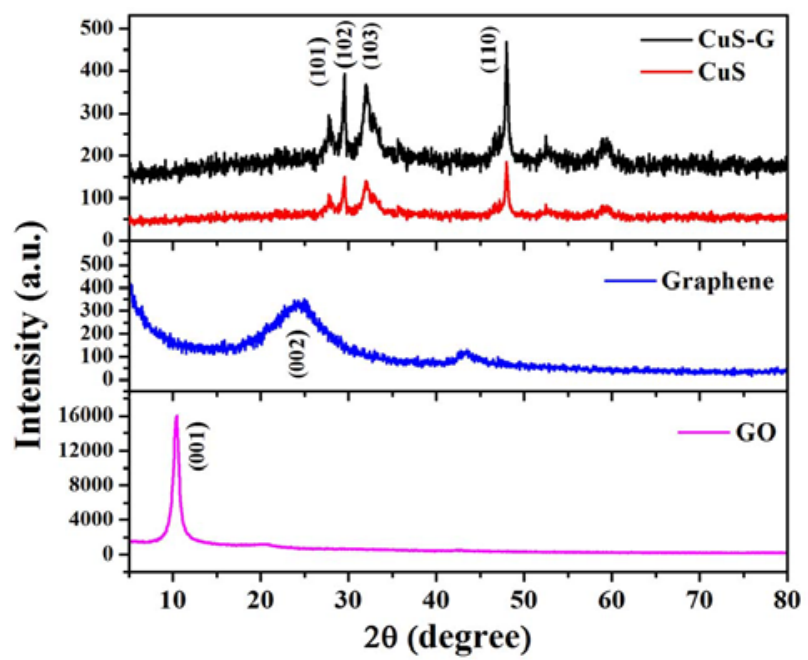

Figure1XRD diffraction patterns of GO, graphene, CuS and CuS-G.

The detailed structure of CuS and CuS-G was shown by SEM and TEM in fig. 2. As can be seen from the images, cubic shape was obtained while solution A was added. With the extending of the reaction time, the mixtures of cubic and hollow cube copper sulfides are appears. When the reaction time of over $24 \mathrm{~h}$, the copper sulfide exhibit amorphous structure. In fig. $2 \mathrm{~b}$, it can be clearly seen that a great number of cube-shaped products were attached onto the carbon sheets. Simultaneously, some of cubic products were inserted into sheets, but not connected to the surface of sheets. According to the report, ${ }^{[19,20]}$, when graphite oxide to grapheme by the chemical conversion, it is prone to holes and defects on the carbon grid. Therefore, the crystal may be grown around these holes and defects, and they tend to be inserted into the sheets ${ }^{[21]}$. Base on previous studies about the formation mechanism, ${ }^{[16,22]}$, we surmised the possible formation mechanism of CuS-G. Firstly, $\mathrm{Cu}^{2+}$ and EDTA can form $\mathrm{Cu}^{2+}$-EDTA; then the formed $\mathrm{Cu}^{2+}$-EDTA can transfer to the GO sheets via the intercalation or adsorption. In addition, the glucose can be used as the reducing agent. In the reaction process, $\mathrm{Cu}^{2+}$ can be reduced to $\mathrm{Cu}^{+}$in the presence of glucose, forming $\mathrm{Cu}_{2} \mathrm{O}$ crystals. At 
the same time, $\mathrm{H}_{2} \mathrm{~S}$ can be generated by $\mathrm{CS}_{2}$ and ethylenediamine in the solution A. After reacting with $\mathrm{H}_{2} \mathrm{~S}$, the $\mathrm{Cu}_{2} \mathrm{O}$ is converted into $\mathrm{CuS}$ crystals. Moreover, throughout the reaction, GO is reduced to graphene.
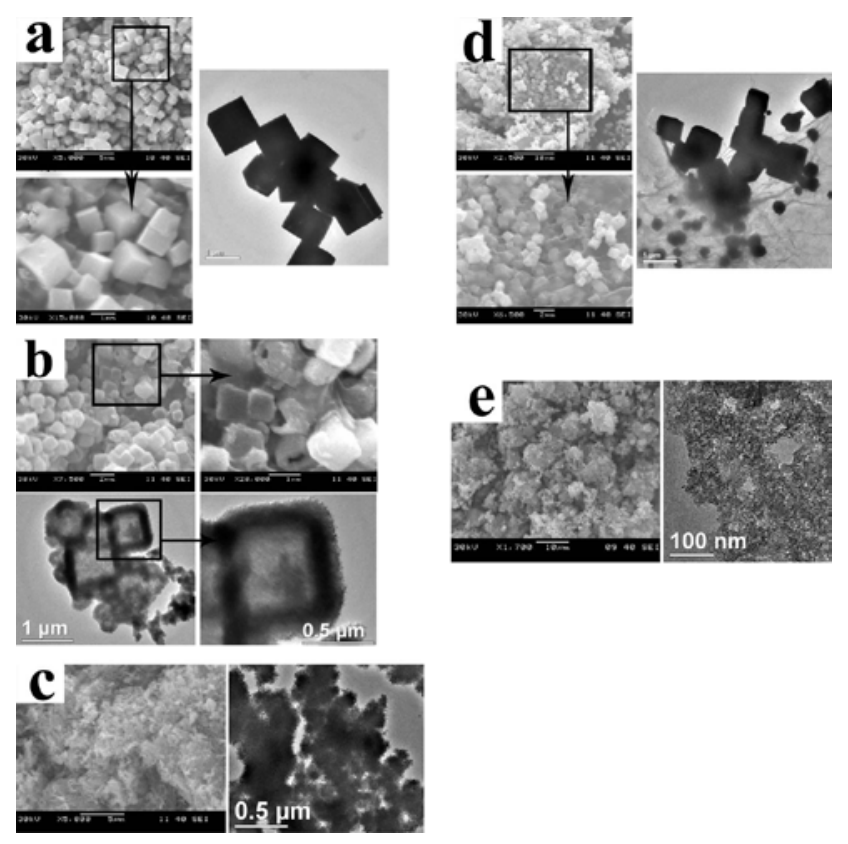

Figure2 Typical SEM and TEM images of (a) $\mathrm{Cu}_{2} \mathrm{O}$, (b) $\mathrm{CuS}$ (after $1 \mathrm{~h}$ the solution $\mathrm{A}$ was added), (c) CuS 5h (after 5h the solution A was added), (d) $\mathrm{Cu}_{2} \mathrm{O}-\mathrm{G}$, (e) $\mathrm{CuS}-\mathrm{G}$ (after $1 \mathrm{~h}$ the solution A was added)

FTIR is employed to better observe the chemical structure of GO, CuS-G composite. In fig. 3, the characteristic peaks of GO are the absorption bands corresponding to epoxy $\mathrm{C}-\mathrm{O}$ stretching vibrations at $1042 \mathrm{~cm}^{-1}$, O-H deformation vibrations of tertiary $\mathrm{C}-\mathrm{OH}$ at $1390 \mathrm{~cm}^{-1}$, carboxy O-H deformation vibrations at $1622 \mathrm{~cm}^{-1}$, and carboxy $\mathrm{C}=\mathrm{O}$ stretching vibrations at $1729 \mathrm{~cm}^{-1}$. However, the peaks for the oxygen-containing functional groups after the reaction are reduced significantly. Moreover, for CuS-G, the adsorption peak around $1566 \mathrm{~cm}^{-1}$ may be attributed to the unoxidized carbon backbone stretching vibrations of graphene ${ }^{[17,23]}$. Simultaneously, the absorption peak at the $940 \mathrm{~cm}^{-1}$ associated with $\mathrm{Cu}-\mathrm{S}$ vibration. The band at $1081 \mathrm{~cm}^{-1}$ corresponding to $\mathrm{C}-\mathrm{H}$ bending frequencies. In addition, the composite can be further testified by XPS data (fig. 4). In XPS spectra, the binding energy at $937.3 \mathrm{eV}$ and $162.1 \mathrm{eV}$ is assigned to $\mathrm{Cu} 2 \mathrm{p}_{3 / 2}$ and $\mathrm{S} 2 \mathrm{p}_{1 / 2}$, respectively.
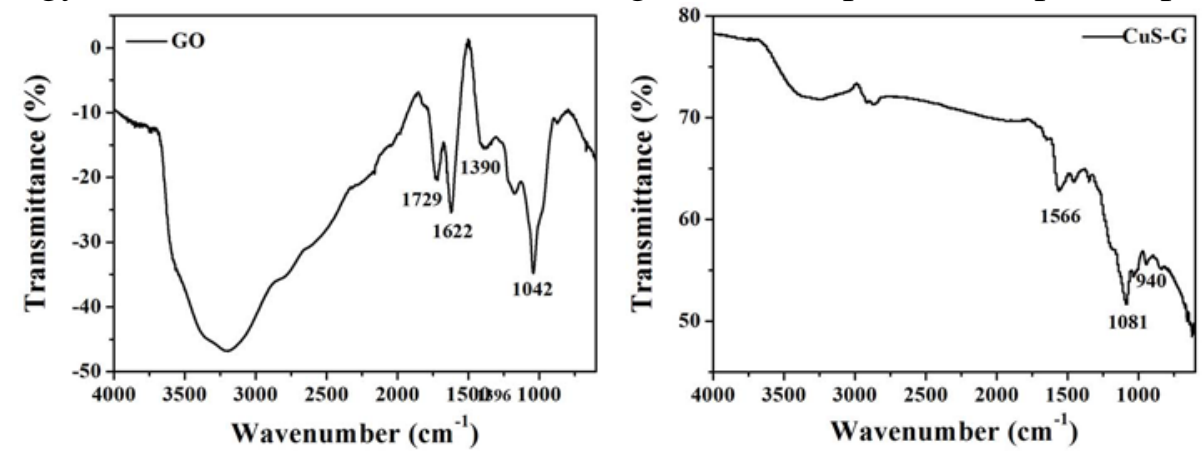

Figure3 FTIR spectra of GO and CuS-G 

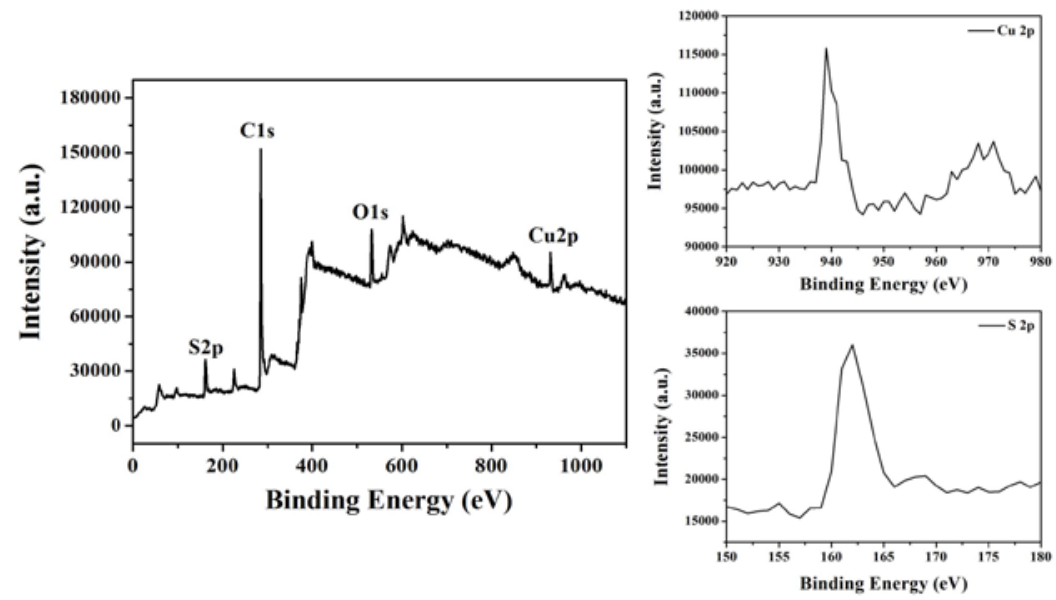

Figure4 XPS wide-scan spectrum of CuS-G composite and high-resolution XPS spectra of Cu 2p, S $2 \mathrm{p}$

\section{Conclusions}

In conclusion, CuS were deposited onto graphene sheets by a mild experimental condition. Experimental results indicate that graphene oxide was reduced to graphene with the formation of CuS.

\section{Acknowledgements}

This work was supported by the Fundamental Research Funds for Changzhou Vocational Institute of Light Industry.

\section{References}

[1] Banerjee, S., Bhattacharya, S. \& Chakravorty, D.,Resistivity hysteresis of Ag2S nanocomposites. The Journal of Physical Chemistry C,111(36), pp. 13410-13413, 2007.

[2] Shalom, M., Rühle, Sven., Hod, I., Yahav, S. \& Zaban, A., Energy level alignment in CdS quantum dot sensitized solar cells using molecular dipoles.Journal of the American Chemical Society, 131(29), pp. 9876-9877, 2009.

[3] Yu, J., Zhang, J. \&Liu, S., Ion-exchange synthesis and enhanced visible-light photoactivity of CuS/ZnS nanocomposite hollow spheres.The Journal of Physical Chemistry C, 114(32), pp. 13642-13649, 2010.

[4] Roy, P. \& Srivastava, S.K.,Hydrothermal growth of CuS nanowires from Cu-dithiooxamide, a novel single-source precursor.Crystal Growth \& Design, 6(8), pp. 1921-1926, 2006.

[5] Li, F., Bi, W.T., Kong, T. \& Qin, Q.H., Optical,photocatalytic properties of novel CuS nanoplate-based architectures synthesised by a solvothermal route. Crystal Research and Technology, 44(7), pp. 729-735, 2009.

[6] Yang, Z.H., Zhang, D.P., Zhang, W.X. \& Chen, M., Controlled synthesis of cuprous oxide nanospheres and copper sulfide hollow nanospheres.Journal of Physics and Chemistry of Solids, 70(5), PP. 840-846, 2009.

[7] Lai, C.X., Wu, Q.B., Chen, J., Wen, L.S. \& Ren, S., Large-area aligned branched Cu2S nanostructure arrays: room-temperature synthesis and growth mechanism. Nanotechnology, 21 (21), pp. 215602 (1-5), 2010. 
[8] Zhai, B. G., Yang, L. \& Huang, Y. M., ZnO nanorods decorated graphene/ZnO nanoparticle composite as the counter electrode of dye-sensitised solar cells. Materials Research Innovations, 19, pp. S7 (15-20), 2015.

[9] Xu, B.F., Lin, Z.D., Du, C.M., Lin, H.B., Liang, K.Y., Qiu, W.P. \& Yang. G.L., Mechanical properties, morphology and thermal conductivity of polyamide composites filled with graphene nanoplatelets, Al2O3 and graphite. Materials Research Innovations, 19, pp. S1 (388-391), 2015.

[10] Kim, K.S., Zhao, Y., Jang, H., Lee, S.Y., Kim, J.M., Kim, K.S., Ahn, J.H., Kim, P., Choi, J. Y. \& Hong, B.H., Large-Scale Pattern Growth of Graphene Films for Stretchable Transparent Electrodes. Nature, 457, pp. 706-710, 2009.

[11] Pan, S.G., Zhu, J.W. \& Liu, X.H., Preparation, electrochemical properties, and adsorption kinetics of Ni3S2/graphene nanocomposites using alkyldithiocarbonatio complexes of nickel(II) as single-source precursors. New Journal of Chemistry, 37(3), pp. 654-662, 2013.

[12] Pan, S.G. \& Liu, X.H., CdS-Graphene nanocomposite: synthesis, adsorption kinetics and high photocatalytic performance under visible light irradiation. New Journal of Chemistry, 36(3), pp. 1781-1787, 2012.

[13] Pan, S.G. \& Liu, X.H., ZnS-Graphene nanocomposite: synthesis, characterization and optical properties. Journal of Solid State Chemistry, 191, pp. 51-56, 2012.

[14] Pan, S.G., Liu, X.H. \& Wang, X., Preparation of Ag2S-Graphene Nanocomposite from a single sourceprecursor and its surface-enhanced Raman scattering and photoluminescent activity. Materials Characterization,62(11), pp. 1094-1101, 2011.

[15] Hummers, W.S. \& Offeman, R.E., Preparation of graphitic oxide. Journal of the American Chemical Society, 80(6), pp. 1339, 1958.

[16] Basu, M., Sinha, A.K., Pradhan, M., Sarkar, S., Govind. \& Pal, Tarasankar., CuO barrier limited corrosion of solid Cu2O leading to preferential transport of $\mathrm{Cu}$ (I) ion for hollow Cu7S4 cube formation. The Journal of Physical Chemistry C, 115(25), pp. 12275-12282, 2011.

[17] Nethravathi, C., Nishaa, T., Ravishankar, N. Shivakumarac, C. \& Rajamathi, M., Graphene-nanocrystalline metal sulphide composites produced by a one-pot reaction starting from graphite oxide Carbon, 47(8), pp. 2054-2059, 2009.

[18] Cai, D. Y. \& Song, M., Preparation of fully exfoliated graphite oxide nanoplatelets in organic solvents. Journal of Materials Chemistry, 17(35), pp. 3678-3680, 2007.

[19] Kudin, K.N., Ozbas, B., Schniepp, H.C., Prud'homme, R.K., Aksay, I.A. \& R, Car., Raman spectra of graphite oxide and functionalized graphene sheets. Nano Letteers, 8(1), pp. 36-41, 2008.

[20] McAllister, M.J., Li, J.L., Adamson, D.H., Schniepp, H.C., Abdala, A.A., Liu, J. \& Aksay, I.A., Single sheet functionalized graphene by oxidation and thermal expansion of graphite. Chemistry of Materials, 19(18), pp. 4396-4404, 2007.

[21] Yu, Y., Ma, L.L., Huang, W.Y., Du, F,P., Yu, J.C. \& Yu J G., Sonication assisted deposition of Cu2U nanoparticles on multiwall carbon nanotubes with polyol process. Carbon, 43, pp. 670-673, 2005.

[22] Fu, Y.S. \& Wang, X., Magnetically separable ZnFe2O4-graphene catalyst and its high photocatalytic performance under visible light irradiation. Industrial \& Engineering Chemistry Research, 50(12), pp. 7210-7218, 2011.

[23] Jeong, H.K., Lee, Y.P., Lahaye, R.J.W.E., Park, M.H., An, K.H., Kim, I.J., Yang, C.W., Park, C.Y., Ruoff, R.S. \& Lee, Y.H., Evidence of graphitic AB stacking order of graphite oxides.Journal of the American Chemical Society, 130(4), pp. 1362-1366, 2008 